\title{
Braided Rope Sensor Based on Carbon Nanotube Yarn
}

\author{
Yunfei Bai, Qi Xia, Jianghan Feng, Han Liu, Jialin Zheng, Danyao Xu, Sidra Saleemi and Fujun Xu* \\ College of Textiles, Donghua University, China
}

*Corresponding author: Fujun Xu, Associate Professor, College of Textiles, Donghua University, 2999 North Renmin Road, Songjiang District, Shanghai, 201620 PR, China.

Received Date: July 15, 2019

Published Date: July 26, 2019

\begin{abstract}
Carbon nanotube (CNT) is exhibited outstanding electrical, mechanical, and structural properties. CNT yarn is widely used in textile structures as multifunctional yarn and is suitable as strain sensor. Here, use two methods for braiding CNT yarn into rope to make sensing ropes. The properties of CNT yarn and different ropes were tested, then according to the result the best method was chosen for the preparation of sensing rope. Results show the CNT yarn have enough strength to be used in braiding process, CNT embedded rope structure has more stable sensing property than CNT braided rope structure. The rope has approximately $\geq 7 \%$ resistance change ratio per $10 \%$ strain, so the embedded rope structure is the optimal structure for sensing rope. The sensing rope will help to improve the development of strain sensor in textile structure.
\end{abstract}

\section{Introduction}

Carbon nanotube (CNT) is exhibited outstanding electrical, mechanical, and structural properties, and proved to be an extremely promising candidate for various applications in material science [1-5]. At present, there are three commonly used methods for the preparation of CNT yarns: direct spinning, solution spinning and carbon nanotube array spinning. Carbon nanotube array spinning is the better method to prepare CNT yarns currently [6].

Carbon nanotube yarns are widely used in textile structures as multifunctional yarns. They have piezoresistive effect and can be used as strain sensors $[7,8]$, such as nondestructive testing, which are mainly used for crack propagation of composites. Carbon nanotube yarns can be combined with a variety of materials such as bandages, gloves, socks and so on to monitor and prevent. At the same time, carbon nanotube yarns have excellent mechanical and electrical properties, so they can be used in many fields, such as conductive materials, pressure sensing materials, microstrip antennas and so on.

However, CNT yarns have not been used in rope braiding. Ropes usually need to bear huge force, and their unexpected rupture would bring hidden dangers [9]. If CNT yarn strain sensors are used in ropes, the stress situation of the rope would be observed, and rupture caused by unbearable force would be prevented. But there are many limitations in current CNT yarn preparation methods. Single CNT yarns still have some weaknesses, such as minor diameter, poor abrasion resistance, easy to produce too much hairiness in preparation, so it is difficult to realize industrial application [10].

In order to obtain sensing rope, the CNT film was twisted into a yarn and embedded in the rope to achieve the preparation of the rope sensor. With this process, the CNT yarn could have enough diameter and mechanical properties to be braided in ropes. The optimal braiding structure for sensing rope was also discussed.

\section{Experimental Section}

\section{Preparation of CNT yarn}

To make a large-diameter and high-strength CNT yarn, twisting a CNT strip to form a composite yarn. The diameter, twist angle and CNT volume fraction of the yarn are controllable by adjusting the film strip's width and twisting process. In addition, the twisting process provides shear force, which improves the yarn's surface orientation.

Figure 1a shows the schematic diagram of twisting process. Here's the operation steps: Cut the CNT film into strips. Set the rotating speed of the twisting motor. Fix both ends of the strip to prevent untwisting. Then start the twisting machine for yarn preparation. Figure 1b shows the CNT yarn. 


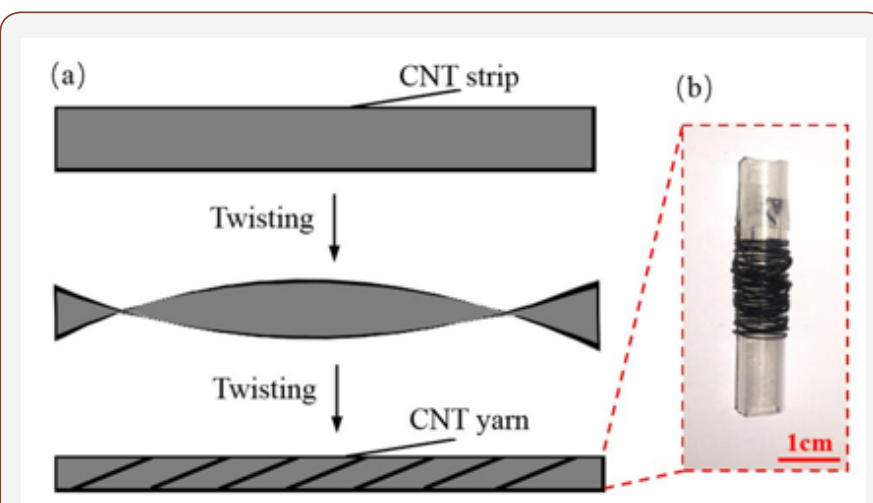

Figure 1: Preparation of CNT yarn (a) the twisting mechanism (b) the CNT yarns.

\section{Braiding of the sensing rope}

There are two main methods to achieve the braided rope with CNT yarn in it: CNT braided process and CNT embedded process.

For the CNT braided process, CNT yarn is interwound cyclically with other common yarns to be braided into the rope. Use a braiding machine (Figure 2a) to prepare the rope. In the process, one of the braiding yarns is CNT yarn and the other are common yarns (Figure $2 \mathrm{~b}$ ). The position of braiding point should be controlled, or the rope would be inhomogeneous. Tension of the braiding yarns needs adjustment during the braiding process, in order to prevent the CNT yarn's elongation. To adjust the tension, pause the machine and rotate the carrier. Figure 2c shows the CNT braided rope.

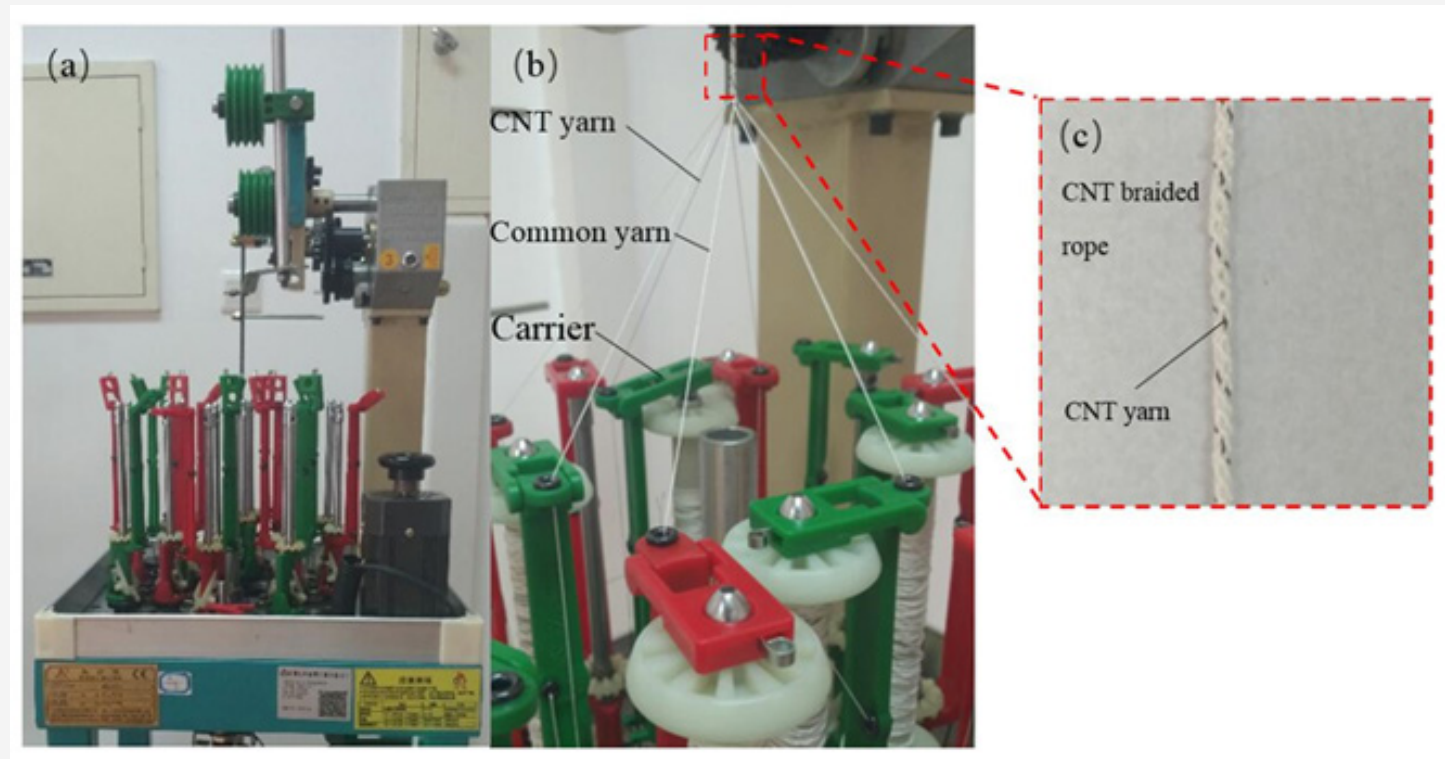

Figure 2: Preparation of CNT braided rope | (a) the 16-carrier braiding machine (b) braiding machine with yarns, use 8 carriers of the machine in the preparation, one is CNT yarn (c) CNT braided rope.

For the CNT embedded process, 8 yarns are braided into a rope as the outer layer and the CNT yarn is embedded into the rope as a core yarn. In the process, put common yarns on the carriers of the braiding machine and CNT yarn as the core (Figure 3a). A pretension on CNT yarn is necessary, in order to keep it straight. The
CNT yarn would be embedded into the rope during the braiding process. To show the inner structure of the rope, use transparent nylon yarn as braiding yarn. Figure 3b shows the CNT embedded rope, it's clear that CNT core yarn is straight in the transparent rope.

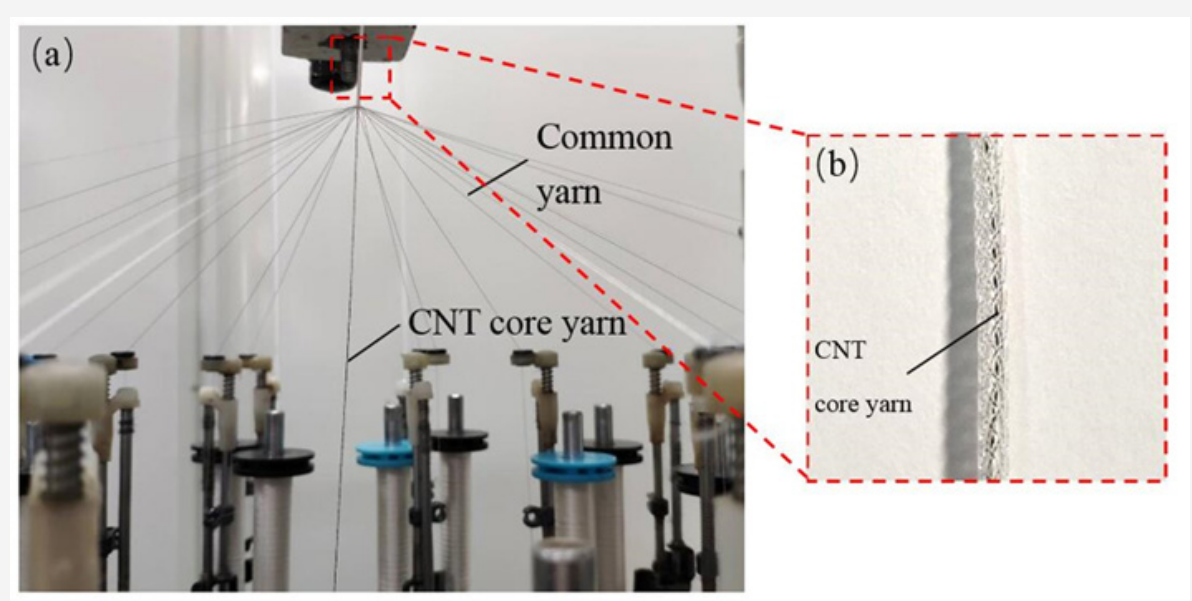

Figure 3: Preparation of CNT embedded rope | (a) braiding machine with yarns, which the CNT yarn as core yarn (b) transparent CNT embedded rope with core yarn inside. 


\section{Results and Discussion}

\section{Structure of the CNT yarn}

With the twist of $6 / \mathrm{cm}$, the CNT yarn is obtained by single untwisted CNT film, which the width is $5 \mathrm{~mm}$. As shown in Figure $4 \mathrm{a}$, the SEM image of the twisted CNT yarn, from which it can be seen obviously that twist and spiral of the CNT yarn. The diameter of the yarn is quite uniform at $220 \mu \mathrm{m}$ and the twist angle of the CNT yarn is about $15^{\circ}$.

After rupture, stress relaxation occurred inside the yarn and fiber in the yarn slipped, thus the CNT yarn is untwisted and converted back into CNT film (Figure 4b). According to the comparison of Figure 4 a with Figure $4 \mathrm{~b}$, the CNT yarn is transformed from uniform cylindrical smooth structure to broad flat structure.
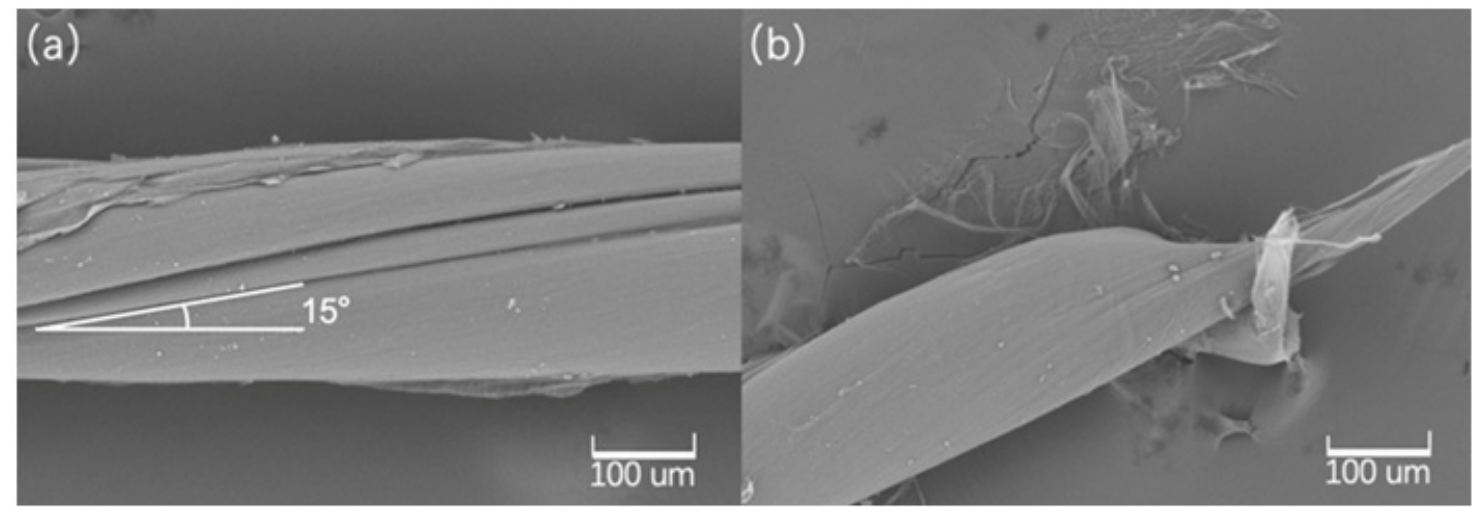

Figure 4: SEM image of the CNT yarn | (a) yarn with obvious twist and spiral figure (b) ruptured CNT yarn, which untwists into flat structure.

\section{Mechanical property of the CNT yarn}

As shown in Figure 5, the maximum strain at fracture of the twisted CNT yarn is $20 \%$, and the stress increases to $80 \mathrm{MPa}$, these values of strength are higher than the values of traditional CNT yarns.

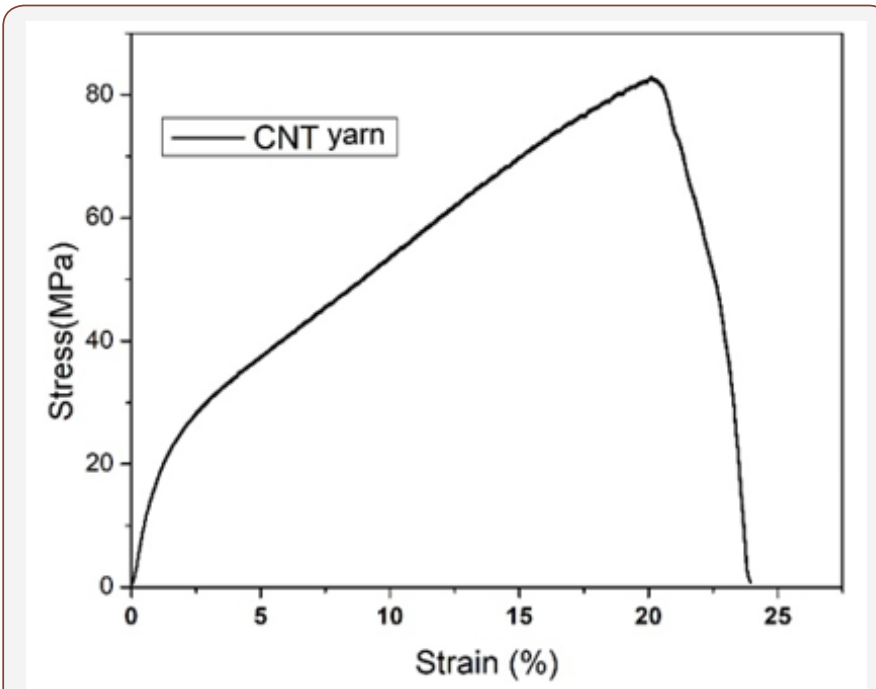

Figure 5: Stress-strain curve of the CNT yarn.

The good mechanical properties of the CNT yarns should be attributed to the action of twisting, which acts in two ways. First, the twisting process fills the internal spaces inside yarn and increases volume content, so the compact structure achieved. Second, the shear force caused by twisting improves the orientation of carbon nanotube arrangement.

Because of the good mechanical properties, this CNT yarn can be used in rope braiding process.

\section{Sensing performance of the CNT yarn}

The resistance change rate of the CNT yarn in the strain range of $35 \%$ is obtained by sensing performance test, in which the Y-axis is the resistance change rate and the $\mathrm{X}$-axis is the strain (Figure 6). The result shows that the curve is smooth, and there is a good correspondence between strain and resistance change ratio, which is about $12 \%$ resistance change per $10 \%$ strain. In the same strain condition, the resistance change ratio of twisted CNT yarn is higher than that of traditional CNT yarn.

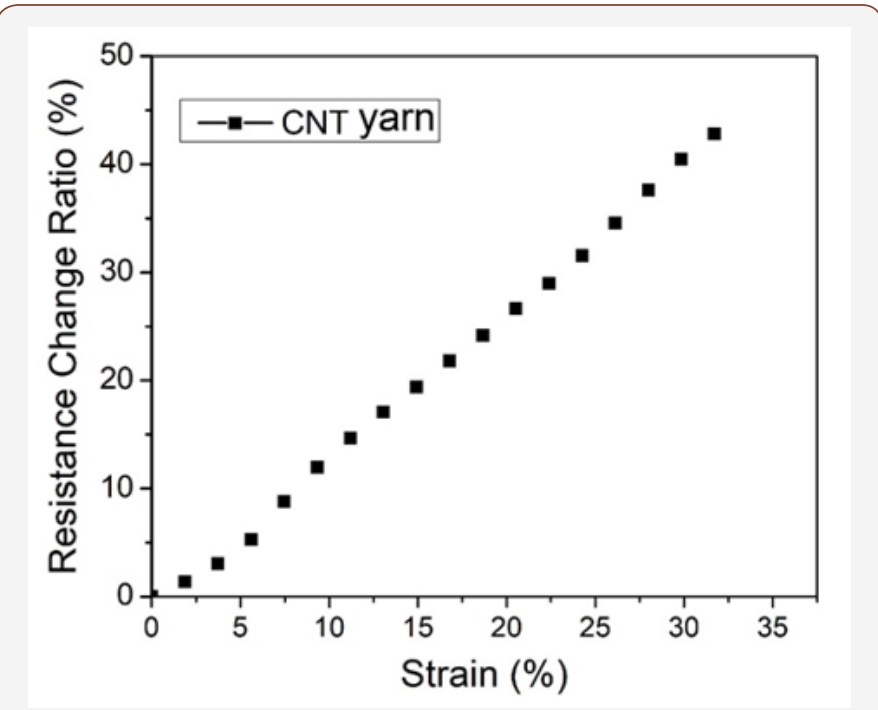

Figure 6: Sensing performance of the CNT yarn.

\section{Optimization of the braiding processes}

The sensing performance of the two different structure ropes was tested. Figure 7a shows the sensing performance of the CNT braided rope: as the strain increases, the resistance change rate fluctuates significantly, and comparing to the CNT yarn, the linearity 
is affected to obvious extent. The main reason is that the carbon nanotube yarn generates buckling during the braiding process, and when the rope is stretched, the CNT yarn itself not only is elongated, but also suffers the pressing effect of the nearby yarns, so the fluctuation is significant. In addition, the sensing performance will be further affected due to the direct exposure of the sensing yarn to the complex application environment.
Figure $7 \mathrm{~b}$ shows the sensing performance of the CNT embedded rope: compared with the CNT braided rope, the rope with the CNT core yarn embedded in it has a moderate fluctuation in the resistance change ratio, the sensing performance is improved because the sensing yarn in the rope keeps straight and is not subjected to obvious buckling. The rope's resistance change ratio is approximately $\geq 7 \%$ per $10 \%$ strain, which is obviously lower than single CNT yarn, but it is enough to reflect the rope's elongation.

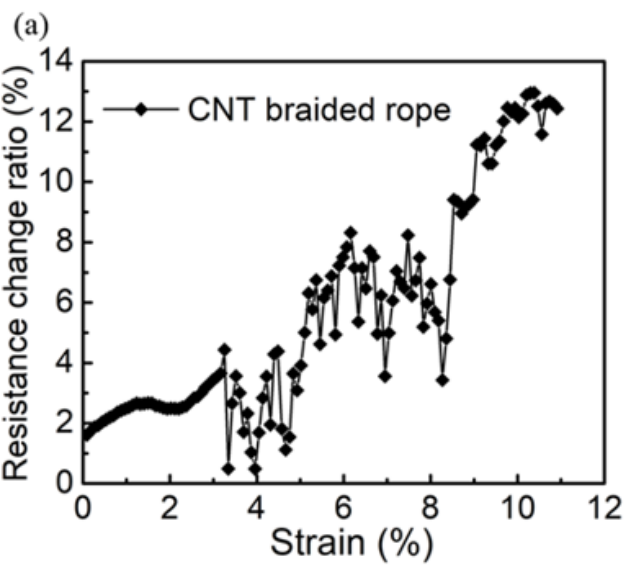

(b)

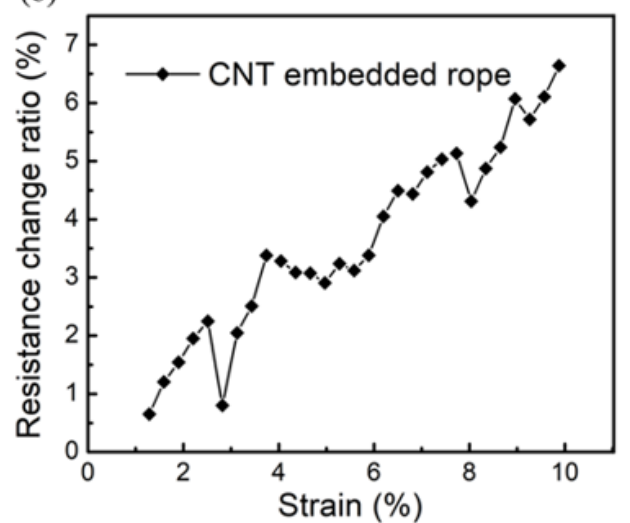

Figure 7: Sensing performance of different structure ropes | (a) sensing performance of CNT braided rope (b) sensing performance of CNT embedded rope.

After comparison between 2 braiding process, it's obvious that CNT embedded structure is the better structure for rope sensor based on CNT yarn.

\section{Conclusion}

In summary, prepared CNT yarn with good properties as strain sensor, then realized the combination of CNT yarn sensor and braiding rope. Compared different braiding process and chose the optimal one. Finally, prepared braided rope sensor based on CNT yarn.

Twisted CNT strip into sensing yarn. Observed the yarn's structure, tested its mechanical properties and sensing performance. The yarn has $220 \mu \mathrm{m}$ diameter, $80 \mathrm{MPa}$ strength and good breaking elongation as $20 \%$. As a result, this CNT yarn is suitable for rope braiding. The sensing performance test showed that there is a good correspondence between its strain and resistance change ratio, which is about $12 \%$ resistance change per $10 \%$ strain. So, it can be used as a strain sensor.

After that, used two different methods to braid rope sensor with CNT yarn and other common yarns, then compared their sensing performance in order to choose an optimal structure for rope sensor. Results showed that CNT embedded rope structure had more stable sensing property than CNT braided rope structure. The rope has approximately $7 \%$ resistance change ratio per $10 \%$ strain, which is enough to observe its elongation, so it's practical to use CNT yarn in braided rope. The sensing rope will help to improve the development of strain sensor in textile structure.

\section{Conflict of Interest}

Authors declare no conflict of interest.

\section{References}

1. (1999) Recent advances in the chemistry and physics of fullerenes and related materials. Kamat KV, Guldi DM, Kadish KM (edts), The Electrochemical Society, USA, 5(97-42): 549-560.

2. Yu MF, Lourie O, Dyer MJ, Moloni K, Kelly TF, et.al. (2000) Strength and breaking mechanism of multiwalled carbon nanotubes under tensile load. Science 287(5453): 637-640.

3. Iijima S (1991) Helical microtubules of graphitic carbon. Nature 354: 56-58.

4. Liu W, Xu F, Zhu N, Wang S (2016) Mechanical and Electrical Properties of Carbon Nanotube/Polydimethylsiloxane Composites Yarn. Journal of Engineered Fibers and Fabrics11(4): 36-42.

5. Oland E, Rune S, Shaun F (2017) Condition monitoring technologies for synthetic fiber ropes-a review. International Journal of Prognostics and Health Management 8(2): 14-15.

6. Frackowiak E, Beguin F (2002) Electrochemical storage of energy in carbon nanotubes and nanostructured carbons. Carbon 40(10): 17751787.

7. Lipomi DJ, Vosgueritchian M, C-K Tee B, Hellstrom SL, Lee JA, et al. (2011) Skin-like pressure and strain sensors based on transparent elastic films of carbon nanotubes. Nature Nanotechnology 6: 788-792.

8. Baughman RH, Zakhidov AA, de Heer WA (2002) Carbon Nanotubes--the Route Toward Applications. Science 297(5582): 787-792.

9. O'donnell HJ, Hoffman TJ, Barker FH (2000) Rope tension monitoring assembly and method: US Patent 6,123,176[P].

10. Baughman RH, Cui C, Zakhidov AA, Iqbal Z, Barisci JN, et al. (1999) Carbon nanotube actuators. Science 284(5418):1340-1344.

\section{Acknowledgement}

None. 